\title{
Seasonal forecast of tropical climate with coupled ocean'atmosphere general circulation models: on the respective role of the atmosphere and the ocean components in the drift of the surface temperature error
}

\author{
A. Lazar, A. Vintzileos, F.J. Doblas-Reyes, P. Rogel \& P. Delecluse
}

To cite this article: A. Lazar, A. Vintzileos, F.J. Doblas-Reyes, P. Rogel \& P. Delecluse (2005) Seasonal forecast of tropical climate with coupled ocean'atmosphere general circulation models: on the respective role of the atmosphere and the ocean components in the drift of the surface temperature error, Tellus A: Dynamic Meteorology and Oceanography, 57:3, 387-397, DOI: 10.3402/tellusa.v57i3.14701

To link to this article: https://doi.org/10.3402/tellusa.v57i3.14701

\section{(c) 2005 The Author(s). Published by Taylor \& Francis.}

\section{曲 Published online: 15 Dec 2016.}

Submit your article to this journal $\square$

\section{Џ Article views: 20}




\title{
Seasonal forecast of tropical climate with coupled ocean-atmosphere general circulation models: on the respective role of the atmosphere and the ocean components in the drift of the surface temperature error
}

\author{
By A. LAZAR ${ }^{1 *}$, A. VINTZILEOS ${ }^{2}$, F. J. DOBLAS-REYES ${ }^{3}$, P. ROGEL $^{4}$ \\ and P. DELECLUSE ${ }^{5}, \quad{ }^{1}$ LOCEAN, UMR 7617 CNRS/IRD/Université Pierre \& Marie CURIE; 4 pl. Jussieu, \\ 75252 Paris Cedex 05, France; ${ }^{2}$ NOAA/NCEP/EMC, 5200 Auth Road, Camp Springs, MD 20746, USA; ${ }^{3}$ ECMWF, \\ Shinfield Park, Reading RG2 9AX, UK; ${ }^{4}$ CERFACS, 42, Avenue Gaspard Coriolis, 31057 Toulouse Cedex 01, France; \\ ${ }^{5}$ LSCE, Bât. 709, Orme des Merisiers, F-91191 Gif-sur-Yvette Cedex, France
}

(Manuscript received 12 April 2004; in final form 21 January 2005)

\begin{abstract}
Seasonal forecasting based on coupled general circulation models suffers from important errors. In order to provide insight into the causes of these errors in relation to ocean and atmosphere models, we carried out a comparison using a set of 10-yr ensemble hindcasts of four coupled climate models of the DEMETER project. The four models are based on two different atmosphere models and three different ocean models. This allows us to analyse the relative weight of the ocean and atmosphere components in the error of a coupled model. Using the hindcast climatologies over the years 1991 to 2000, we looked specifically at the sea surface and soil level temperature over the tropics with respect to the hindcast start date. Our results indicate that the monthly evolution of large mean deviations from the observations (> $\pm 1{ }^{\circ} \mathrm{C}$ after 6 months) can be decomposed into two terms. One is the first month error, which results from the errors in the initial conditions plus the error introduced by the first month of coupling. It corresponds to the slowly varying component of the error, comparable to an initial shift that persists during the entire coupled experiment. The other term is the remaining time-evolving error, which is fast varying. We show that whereas the slowly varying term is strongly dependent upon the ocean and atmosphere component chosen for the coupling, the atmosphere generally controls the rapidly varying term to first order. The partition appears to be more balanced over some fractions of the Pacific warm pool and the east-equatorial coastal upwelling at certain seasons. These results, specific to seasonal forecasting and probably model dependent, can hardly be interpreted in terms of coupling mechanisms. The weak sensitivity of the mean error to the ocean component could in particular be related to the current limitations of state-of-the-art climate models.
\end{abstract}

\section{Introduction}

The improvement of seasonal weather forecasting is a current scientific challenge. Comprehensive climate models, which are generally accepted as the most promising tool for performing seasonal forecasting, are capable of reproducing important characteristics of the dominant variability modes (e.g. Latif et al., 1993; Ji et al., 1994; Rosati et al., 1997; Kirtman, 1997; Vintzileos et al., 1999a;99b; Guilyardi et al., 2004). The differences and similarities of simulated tropical mean climate and

*Corresponding author.

e-mail: ala@lodyc.jussieu.fr variability have been described in various intercomparison studies of coupled general circulation models (CGCMs) (Mechoso et al., 1995; Delecluse et al., 1998; Latif et al., 2001; AchutaRao and Sperber, 2002; Davey et al., 2002; Schneider et al., 2003). Many of these groups reported that simulated mean climates were drifting, reaching steady states after many years. Unrealistic features of simulated interannual modes, e.g. quasi-bienniality in the periodicity of the El Niño-Southern Oscillation (ENSO) cycle, are most likely related to such climate biases. In spite of these errors, Stockdale (1997) showed that after removing this climate drift $a$ posteriori it was possible to obtain good ENSO forecasts at lead times of up to 6 months (more sophisticated methodologies for taking into account this climate drift have also been presented, 
e.g. Yang and Anderson, 2000). However, it is expected that better models, i.e. with no drifting mean state, should allow better seasonal forecasting. A major task therefore is to achieve a better understanding of how the state-of-the-art CGCMs work, and particularly the nature and causes of the accompanying errors.

The DEMETER project provides a new opportunity to compare retrospective seasonal forecasts, or hindcasts, for several CGCMs over the entire globe. Of particular interest is the fact that of the seven CGCMs, four form two pairs, where each of the pairs is made of a specific atmosphere model coupled to two different ocean models (hereafter, these pairs are called atmosphere model pairs). The atmosphere GCMs (AGCMs) are IFS and ARPEGE, and the ocean GCMs (OGCMs) are ORCA, OPA8 and HOPE (see the DEMETER project description in Palmer et al., 2004). It is therefore possible to use a given atmosphere model pair to examine the impact on the coupled GCM errors of switching the ocean component while keeping the same atmosphere. Such a switch can reveal the weak or strong sensitivity of given errors to the coupling and to the ocean model, possibly leading to more precise interpretations of their causes.

Using this method, we derive and test the robustness of a formulation of the error in the sea surface temperature and surface air temperature (hereafter indistinctively called surface temperature), as a linear function of a fast-varying component, which is atmosphere dependent, and a slowly varying component which is sensitive to the ocean and atmosphere component of the CGCM. We concentrated our study over the tropics where surface temperatures are expected to influence the most seasonal climate variations. It is important to realize that our analysis is not aimed at deciphering the coupling mechanisms at play in CGCMs or in the real world. It is solely intended to provide information on the causes of certain systematic errors that alter state-of-the-art numerical climate forecasts in the tropics.

\section{Methodology}

For the purpose of improving techniques of seasonal forecasting, the DEMETER experiment gathered seven CGCMs and their respective 6-month ensemble hindcasts repeated every 4 months over several decades ranging from the 1960 s to the present. We selected four of these model simulation sets over the same decade (1991-2000), sharing either the ocean or the atmosphere component. The experiments are CERFACS, LODYC, ECMWF and CNRM laboratory simulations (see Palmer et al., 2004). As seen in Table 1, the use of two AGCMs and three OGCMs allows us to describe the four simulations as two atmosphere model pairs. The first pair is made of the ECMWF atmosphere model, IFS, coupled to the most recent version of the LODYC ocean model ORCA, version 8.2, (hereafter IFS/ORCA) and to the Hamburg Ocean Primitive Equation model HOPE-E (hereafter IFS/HOPE). These two ocean models, albeit both $z$-coordinate models, were independently developed, their differences are hence rather representative of differences among models of this type (here in particular the vertical resolution, the turbulent mixing schemes, the mixed layer parametrization, etc.). The second pair is made of the Météo-France atmosphere model ARPEGE, coupled to the same last version of the ORCA model and to an older version of this model, named OPA8.0, (hereafter ARPEGE/ORCA and ARPEGE/OPA8). The differences between these two versions are in fact also large since they use different lateral mixing parametrizations, namely isopycnal diffusion and eddy-induced velocities (Gent and McWilliams, 1990) in ORCA versus just horizontal diffusion in OPA8.0. Various studies have highlighted the major impact of such differences for the tropics in forced (Lengaigne et al., 2003) and coupled modes (Raynaud et al., 2000). This impact can be seen, for example, as one of the consequences of modification of the equatorial stratification related to changes in the mixing formulation.

It is important to note that none of the GCMs uses data assimilation or flux correction and they are consequently comparable in this respect. The models have been initialized as explained in Palmer et al. (2004). Specifically, atmospheric and land-surface initial conditions are the same for all atmosphere components and are taken directly from ERA-40. The ocean initial state was taken from ocean-only simulations forced by ERA-40 fluxes, with a strong relaxation to observed sea surface temperatures (SSTs). However, apart from the use of different relaxation coefficients, the ensembles were created following almost the same strategies. ECMWF and CNRM applied wind stress perturbations to create three (two perturbed and one unperturbed) continuous slightly different ocean-only simulations, from which the

Table 1. Description of the simulations in terms of atmosphere and ocean components

\begin{tabular}{|c|c|c|c|c|}
\hline & $\begin{array}{c}\text { Météo-France } \\
\text { (ARPEGE/OPA8) }\end{array}$ & $\begin{array}{c}\text { CERFACS } \\
\text { (ARPEGE/ORCA) }\end{array}$ & $\begin{array}{c}\text { LODYC } \\
\text { (IFS/ORCA) }\end{array}$ & $\begin{array}{l}\text { ECMWF } \\
\text { (IFS/HOPE) }\end{array}$ \\
\hline Atmosphere component & ARPEGE & ARPEGE & IFS & IFS \\
\hline \multirow[t]{2}{*}{ Resolution } & T63 & T63 & T95 & T95 \\
\hline & 31 levels & 31 levels & 40 levels & 40 levels \\
\hline Ocean component & OPA 8 & ORCA & ORCA & HOPE-E \\
\hline \multirow[t]{2}{*}{ Resolution } & $2.0^{\circ} \times\left(0.5^{\circ}-2.0^{\circ}\right)$ & $2.0^{\circ} \times\left(0.5^{\circ}-2.0^{\circ}\right)$ & $2.0^{\circ} \times\left(0.5^{\circ}-2.0^{\circ}\right)$ & $1.4^{\circ} \times\left(0.3^{\circ}-1.4^{\circ}\right)$ \\
\hline & 31 levels & 31 levels & 31 levels & 29 levels \\
\hline
\end{tabular}


nine-member ensemble was constructed by instantaneously applying (at the starting time) a set of four SST perturbations. LODYC and CERFACS used exactly the same set of ocean initial conditions. These initial conditions were taken from an ensemble of three ocean-only simulations initialized every 3 months (right after the previous start date) from the previous unperturbed ocean simulation, two of them perturbed by wind stress anomalies. The SST perturbations were applied a few days before the start date. As a result, initial ocean conditions shall be considered as potential causes of differences among CGCM surface temperature climatologies. We therefore consider in the following that an ocean or atmosphere "component" corresponds to the circulation model per se plus its initial conditions. Finally, differences arising from the land surface parametrizations, ice-related processes and detailed coupling procedures can also generate variations among hindcasts. They also need to be considered, even though the parametrizations of the land surface processes are likely to have a much greater impact than the two other sources of differences. Consequently, the imperfect nature of the present intercomparison leads us to emphasize the similarities among hindcasts instead of the differences. Indeed, when some similarities are observed amongst surface temperature behaviour for two different CGCMs, it indicates that the differences in the experimental configurations have a negligible impact compared with the similarities in the configurations.

It is necessary to say that various key climate variables and indices have been compared and presented on the DEMETER website (http://www.ecmwf.int/research/demeter/verification). Though verification underlines differences between models, it also indicates that all four models perform at roughly the same level of skill for hindcasting tropical seasonal climate variations on a seasonal scale. This allows us to consider the four CGCMs as appropriate tools of analysis of the quality of state-of-theart numerical climate prediction models. Prior to the following analysis, all the surface temperature fields are averaged over the nine ensemble members, and the resulting ensemble mean forecasts are averaged over the 10 -yr period with respect to the four hindcast start dates (1 February, 1 May, 1 August, 1 November, hereafter SD1, SD2, SD3, SD4 respectively). As an example, the climatology of SD1 corresponds to the ensemble mean of the 6-monthly averages centred on the 15 th of each month, starting in February and ending in July.

\section{Analysis of the errors of the CGCM surface temperature climatologies}

\subsection{Error in equatorial basin averages: the rapid drift and the near-steady components}

Figure 1 displays the time evolution of the error of the surface temperature climatologies for the four starting times, averaged over each of the three equatorial basins between $5^{\circ} \mathrm{N}$ and $5^{\circ} \mathrm{S}$. A striking pattern for all the basins consists of errors of the same

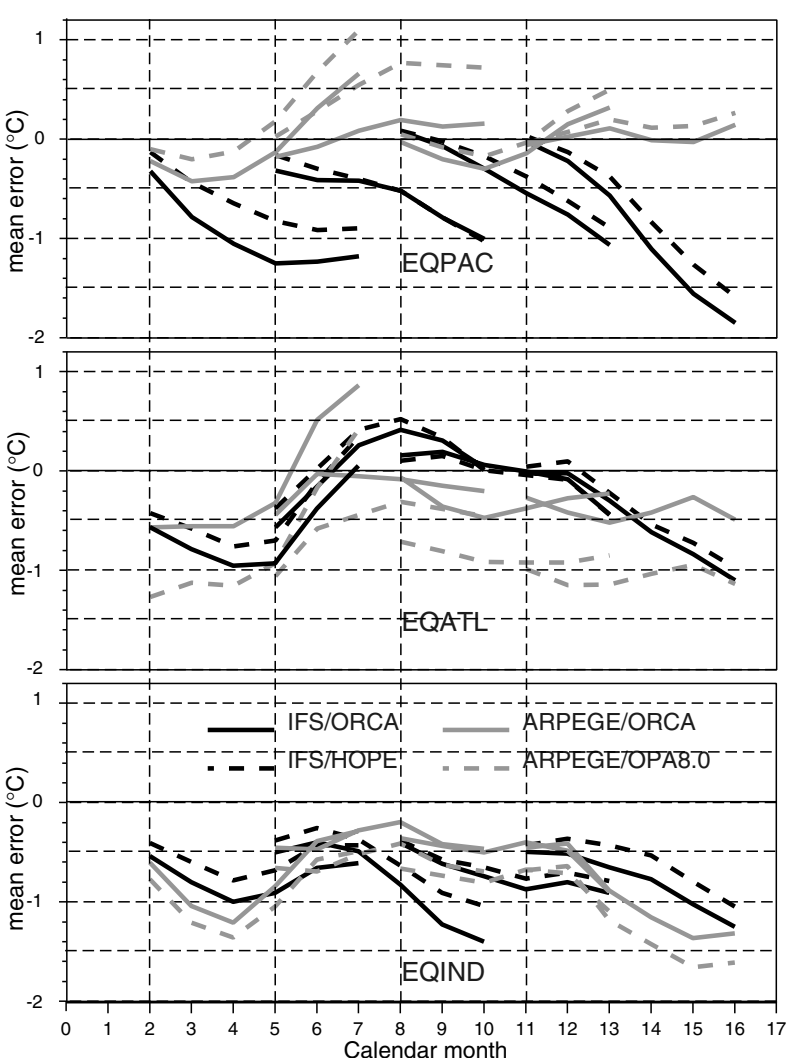

Fig. 1. The mean drift of the equatorial sea surface temperature as a function of time. Geographical averages computed between $5{ }^{\circ} \mathrm{N}$ and 5 ${ }^{\circ} \mathrm{S}$ for the Pacific (130E-80W), Atlantic (70W-30E) and Indian (40E-120E) basins.

atmosphere model (same shading) evolving in close parallel as pairs, separated by a relatively constant gap within a given season. The monthly time derivatives of the error are very similar between two coupled models with the same atmosphere model, regardless of the ocean model. Conversely, the monthly time derivative changes significantly when the atmosphere model is switched to another one. Thus the two hindcasts of the ARPEGE atmosphere model pair tend systematically to drift in parallel towards warmer than observed temperatures in the Pacific, and the two hindcasts of the IFS atmosphere model pair tend in parallel towards colder than observed temperatures. In the Atlantic and the Indian basins, the differences between the two types of time evolution of the two atmosphere model pairs are less dramatic. They differ clearly anyway, and for a given atmosphere model pair the two CGCMs are evolving almost perfectly in parallel. This result indicates that the shape of the error curve is extremely sensitive to the atmosphere component, and much less affected by an ocean component switch, which tends more simply to shift the curve without changing its shape. In other words, the choice of the atmosphere model of a given CGCM appears to condition to first order the temporal variations of the error in equatorial surface temperature. 
The offset that appears to separate the two climatologies of a model pair with a specific atmosphere is also an important characteristic of the error. It is often settled by the first month and evolves weakly. It is generally of the same sign for the climatologies of a given atmosphere model pair and a given basin, regardless of the season. For the pair associated with the IFS atmosphere model, the offset has an average value of about $0.1{ }^{\circ} \mathrm{C}$ and a constant sign. For the ARPEGE model pair, the Pacific basin has an opposite offset to those of the Indian and Atlantic basins. These offsets are only due to changes in the ocean component (same atmosphere model and atmosphere initial conditions). More precisely, two ocean factors can be considered: the differences in the physics of the ocean models or some systematic differences in the ocean initial conditions introduced by the way in which perturbed ocean initial conditions have been constructed. Hence, the choice of the ocean component (i.e. the ocean model and ocean initial conditions) of a given CGCM appears to first order to change an almost constant component of the mean error in the surface temperature.

In order to summarize the results suggested by Fig. 1, it is convenient to decompose the equatorial average of the mean error in surface temperature (ME) as follows:

$\operatorname{ME}(t, \operatorname{atm}$, oce $)=\operatorname{Drift}(t, \operatorname{atm})+\operatorname{ME}_{\text {month1 }}($ oce, atm $)$

where $t$ indicates the time dependency and atm and oce indicate upon which component of the coupled model, ocean or atmosphere, a given term is mostly dependent. The first term on the right-hand side represents the time-varying component of the mean error and is computed as the difference between the mean error at time $t$ and its value for the first month $(\operatorname{Drift}(t)=$ $\left.\operatorname{ME}(t)-\mathrm{ME}_{\text {month } 1}\right)$. The second term is the mean error for the first month. As an example, let us consider the Pacific climatologies for SD1. The deviations relative to month 1 reach about $-1{ }^{\circ} \mathrm{C}$ after 6 months for the two IFS-based CGCMs, and about $+1{ }^{\circ} \mathrm{C}$ for the two ARPEGE-based CGCMs. They are hence essentially dependent on the atmosphere model. On the other hand the month 1 mean error, $\mathrm{ME}_{\text {month } 1}$, takes values of about $-0.1{ }^{\circ} \mathrm{C}$ and $-0.3{ }^{\circ} \mathrm{C}$ for the IFS/ORCA and IFS/HOPE models respectively, and values of $-0.2{ }^{\circ} \mathrm{C}$ and $-0.1{ }^{\circ} \mathrm{C}$ for the ARPEGE/ORCA and ARPEGE/OPA8 models, respectively. These values indicate that the term $\mathrm{ME}_{\text {month } 1}$ in eq. (1) depends on the ocean component, and also on the atmosphere model since it varies between the two CGCMs with the same ORCA ocean component.

Regarding the implications of eq. (1) for the improvement of seasonal predictions in terms of improvement in seasonal forecasting, this decomposition of the error highlights the difference between fast-varying, mainly atmospheric, processes (first term on the right-hand side), and slowly varying, oceanic and possibly atmospheric, mechanisms (second term on the right-hand side). This is of course a well-known decomposition in climate physics, and this set of experiments has the advantage of providing new evidence that it is also valid for interpreting climatological er- rors in seasonal climate forecasts. Hence, let us consider first the example of the equatorial Pacific time-series for the two IFSbased CGCMs in Fig. 1. Using the terminology of eq. (1), it is clearly a case of a rapidly growing drift term but a small mean error for the first month. To improve the forecasts in this region, therefore, the amplitude of the drift must be reduced as a priority. Equation (1) then suggests that one should test some modifications of the physics of the atmosphere model, upon which the term $\operatorname{Drift}(t$, atm $)$ depends, rather than some changes in the ocean component. According to the figure, modifications of the ocean component would in fact only shift the mean error not stabilize it. Conversely, the equatorial Atlantic climatologies of the two ARPEGE-based CGCMs for the SD3 and SD4 start dates come into the category of a rather steady mean error due to a large initial error. An improvement would then require a mere positive shift of the mean error. The strategy suggested by eq. (1) consists of modifying the ocean component, which would change the error for the first month and not the (valuable) steadiness of the error (which is controlled by the atmosphere model). For the ARPEGE/ORCA case, the figure shows that the alternative strategy (a modification of the atmosphere model) would also have worked out for SD3 because the IFS/ORCA climatology is overall better than the ARPEGE/ORCA one. However, it would have been a fruitless choice for SD4 because strong growth is introduced when substituting the IFS model for the ARPEGE model.

It is important to state here that the influence of the atmosphere model on the fast-varying component of the error $\operatorname{Drift}(t, \operatorname{atm})$ does not mean that coupled processes are not involved. We believe instead that coupling can be very active in equatorial basins, particularly in the eastern part of the Atlantic and the Pacific basins. In our opinion, the weak sensitivity of $\operatorname{Drift}(t, \mathrm{~atm})$ to the ocean component would in fact only imply that whatever the fast-varying processes acting on $\operatorname{Drift}(t$, atm $)$ at the equator, coupled and non-coupled, their numerical simulation at intraseasonal frequency is little affected by changes in the ocean component. This could be explained by the current differences between state-of-the-art OGCMs in representing physical mechanisms relevant to these timescales and regions. These differences could either be too small compared with the differences between current AGCMs, or have a large impact at lower frequencies (e.g. parametrization of slow diffusive processes) and be negligible relative to fast-evolving atmospheric processes (e.g. parametrization of convection that is crucial to numerous tropical climate mechanisms).

\subsection{Large-scale patterns of the drift term and their dependence upon the atmosphere model}

It is likely that the average equatorial results discussed above are dominated by a limited number of small areas with stronger than average error. It is therefore necessary to examine horizontal maps of the drift, and we chose to limit our study to its 

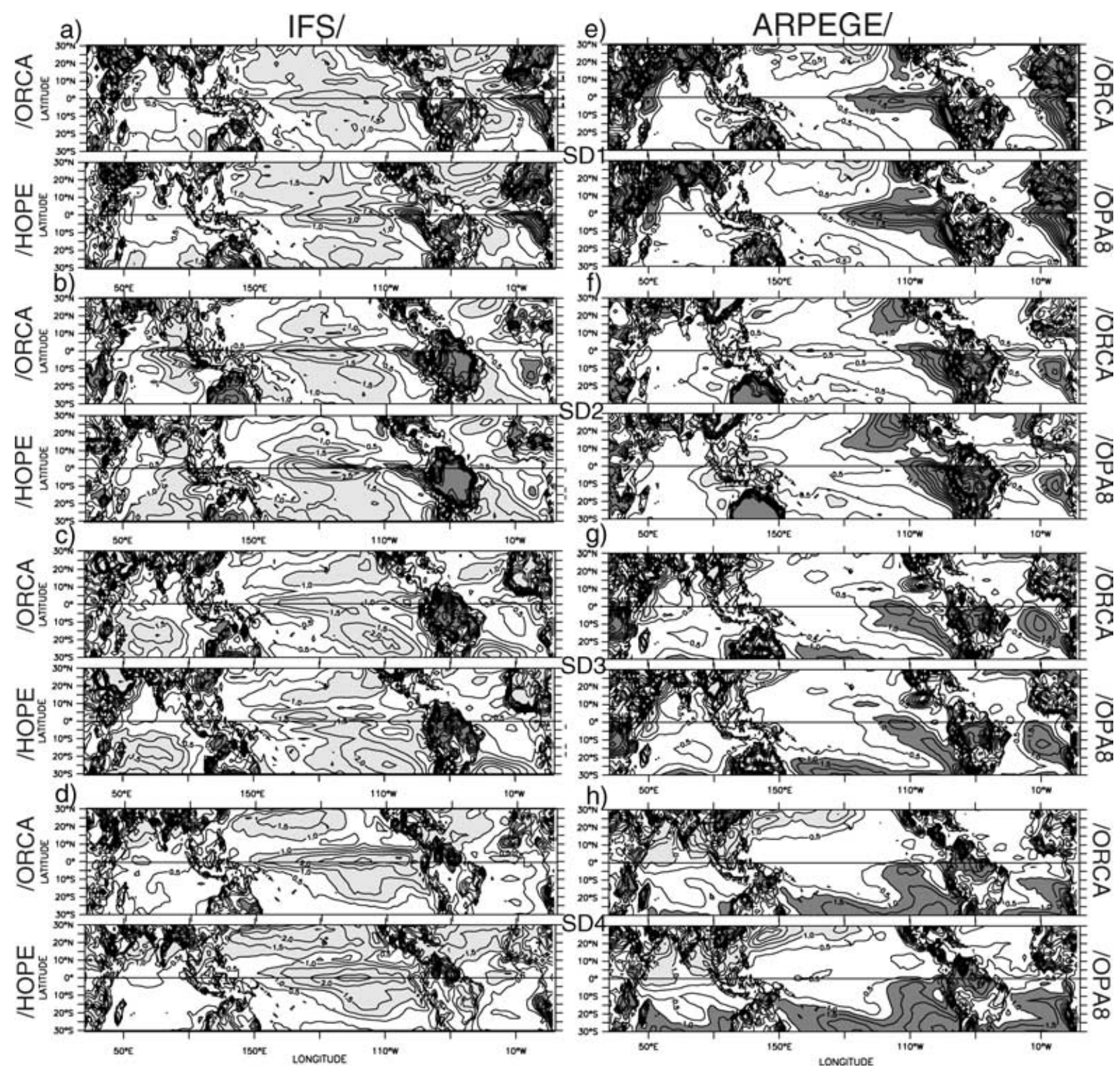

Fig. 2. The mean drift of the tropical surface temperatures as a function of the hindcast starting date, represented by the drift term (month 6 minus month 1, see text for details) computed, for each starting date, for IFS/ORCA and IFS/HOPE (respectively upper and lower subpanel, left column); and for ARPEGE/ORCA and ARPEGE/OPA8 (respectively upper and lower panel, right column). Shading for absolute values larger than $1{ }^{\circ} \mathrm{C}$, dark shading corresponds to positive values, light shading to negative values, contour interval $0.5^{\circ} \mathrm{C}$ up to $5{ }^{\circ} \mathrm{C}$.

patterns after 6 months, computed by subtracting the mean error for the first month from that for the sixth month. In the rest of this paper, "drift term" always refers to the error drift computed this way - it is the term $\operatorname{Drift}(t=6$ months, atm) in eq. (1). Figure 2 presents a geographical comparison of this drift term, with respect to hindcast start date.

Drift term amplitudes greater than $1{ }^{\circ} \mathrm{C}$ are shaded in Fig. 2 since moderate drift values are of less concern in this paper, which seeks a means to refine strategies for improving weather climate forecasting. From a global point of view, it is first striking to realize how well the patterns over the whole tropics appear to match the results discussed in the previous section: climatologies, for a given start date, have a very comparable drift term when sharing the same atmosphere model, and a very different one otherwise. Note also that the comparison of the two atmosphere model pairs indicates that the drift signs and patterns are different, but the amplitudes are comparable. Regarding the two CGCMs using the IFS atmosphere model, the equatorial oceanic regions of most rapid cold drifts are the whole central Pacific $\left(-1\right.$ to $-2{ }^{\circ} \mathrm{C}$ ), eastern Indian (about $-2{ }^{\circ} \mathrm{C}$ during 
hindcast 2) and the eastern Atlantic ( -1 to $-2{ }^{\circ} \mathrm{C}$ during hindcasts 3 and 4). Large warm drifts occur during the upwelling season in the eastern Pacific and Atlantic (up to $+3{ }^{\circ} \mathrm{C}$ during hindcasts 1 and 2). Within the rest of the tropics, both the central Pacific and Atlantic drift strongly negatively. The largest signals (over $\pm 5^{\circ} \mathrm{C}$ ) occur over each of the main monsoon regions during their respective rainy seasons: tropical South America and Australia, India, southwestern China and West Africa (SD2 and SD3). Regarding the ARPEGE/OPA8 and ARPEGE/ORCA models (Fig. 2, right column), large positive equatorial drifts are visible over the central-eastern Pacific (above $+2{ }^{\circ} \mathrm{C}$, corresponding to the spatial average), eastern Atlantic (up to $+3{ }^{\circ} \mathrm{C}$ ) and western Indian basin, mainly during seasons (springsummer) and places of upwelling. Within the tropics, a large positive drift also occurs over off-equatorial upwelling regions, like the eastern Pacific and Atlantic and the northwestern Indian basins. Over continents, as for the IFS pair, the principal monsoon regions experience massive drifts (larger than $\pm 5^{\circ} \mathrm{C}$ ) during their rainy season. Comparison of the above patterns demonstrates that the large drifts respond much more weakly to a change of the ocean component than to a change of the atmosphere model. This therefore supports the generalization of eq. (1) to the large-scale structure of rapidly growing errors, over most of the whole tropical band, including both oceans and continents.

\subsection{Role of the ocean component on the error: effect of slow coupled processes}

The previous qualitative analyses suggested a largely dominant role of the atmosphere relative to the ocean in the time evolution of rapidly growing error. At this stage of the study, it is thus appropriate to characterize this indication spatially. With our DEMETER subset of models, there is one case of ocean component change for each atmosphere model, while there is only one case of atmosphere component change to study (IFS/ORCA to ARPEGE/ORCA). Since one case would not be demonstrative enough, we are therefore constrained to direct the analysis to the two cases of ocean component change. If eq. (1) is valid, the drift relative to month 1 should not change significantly following a switch of the ocean component. Conversely, if an important modification of this time evolution occurs, it indicates that the climatological error cannot be described as in eq. (1) (the ocean component in the coupling has a rapid effect on the error). As in the previous part, we limited the analysis to the drift between month 6 and 1. A suitable drift change index (hereafter DCI) for a given AGCM coupled to two different ocean models, and for a given start date, is then provided by the point-by-point absolute difference between the two drift terms (one per ocean component), relative to the local mean absolute drift term (the average of the two absolute drift terms):

$$
\begin{aligned}
\mathrm{DCI}_{\mathrm{O}}= & \mid \text { Drift }_{\mathrm{OGCM} 1}-\text { Drift }_{\mathrm{OGCM} 2} \mid \times 2 \\
& /\left(\mid \text { Drift }_{\mathrm{OGCM} 1}|+| \text { Drift }_{\mathrm{OGCM} 2} \mid\right),
\end{aligned}
$$

where, again, each of the Drift terms is the difference between the sixth and first month of a CGCM error, for a given start date and a given atmosphere component. If the $\mathrm{DCI}_{O}$ is small, one can conclude that the drift term has little sensitivity to changes in the ocean component, and consequently that the coupling has no, or slow, effects, in agreement with eq. (1). If the $\mathrm{DCI}_{O}$ is large, it is less appropriate to draw firm conclusions since the set of experiment is not perfectly adapted to the intercomparison. As a matter of fact, for a given atmosphere pair, the details of the coupling procedure (i.e. the SST and flux passing methods) are not necessarily the same, and one cannot rule out the possibility that it generates a large $\mathrm{DCI}_{\mathrm{O}}$. The interpretation of this diagnostic then should be limited to the cases of small drift change, and we shall not consider the patterns of large $\mathrm{DCI}_{\mathrm{O}}$.

A preliminary inspection of the $\mathrm{DCI}_{\mathrm{O}}$ distribution indicates that areas of high value are circumscribed to regions of relatively small drift term (not necessary matching them). An approximate threshold absolute value is $1{ }^{\circ} \mathrm{C}$ for the latter (see the unshaded areas on Fig. 2). Such large differences among moderate drift terms can be interpreted as resulting from a more balanced competition between atmospheric and oceanic processes, both with timescales that are locally comparatively slow. For regions of small to moderate drift term, we conclude therefore that it is not possible to deduce a simple partition of the ocean and atmosphere components in terms of their influence on the time evolution of the mean error. In terms of the strategy to improve forecasts, the choice of the ocean component (recall that it includes ocean initial conditions) influences the mean error as much as that of the atmosphere model. However, such regions are less important than regions of rapidly growing errors for the purpose of improving weather forecasts. Consequently, for the sake of clarity of the figure, we chose to limit the presentation of the $\mathrm{DCI}_{\mathrm{O}}$ to regions of drift term with amplitudes larger than $1{ }^{\circ} \mathrm{C}$. In order to do so, Fig. 3 displays the $\mathrm{DCI}_{\mathrm{O}}$ when at least one of the two drift terms in eq. (2) has an absolute value larger than $1^{\circ} \mathrm{C}$. Corresponding regions still occupy very large fractions of the tropics (shaded areas on Fig. 2 and Fig. 3). For both the IFS and the ARPEGE pair, it appears that the larger coherent patterns of high $\mathrm{DCI}_{O}$ (let us say equal to or greater than 0.5 , i.e. 50 per cent) are found mainly within the subtropics, probably associated with the large ensemble spread characteristic of these latitudes (e.g. Vialard et al., 2004). Within the tropics, more localized signals are seasonally associated with some fractions of the equatorial and eastern off-equatorial upwellings and, in particular for the IFS pair, some of the monsoon continental areas detailed above. In order to better understand the behaviour of the mean error within the regions of high $\mathrm{DCI}_{\mathrm{O}}$, we chose some locations to examine later in more detail (see arrows). Two others will supplement this selection of six sites later in the analysis. We limited it to the equatorial ocean (neglecting continental and tropical areas) since this is probably where the question of the respective influences of the ocean and the atmosphere on the growth of the error is the more critical for climate forecasting. Overall, tropical 


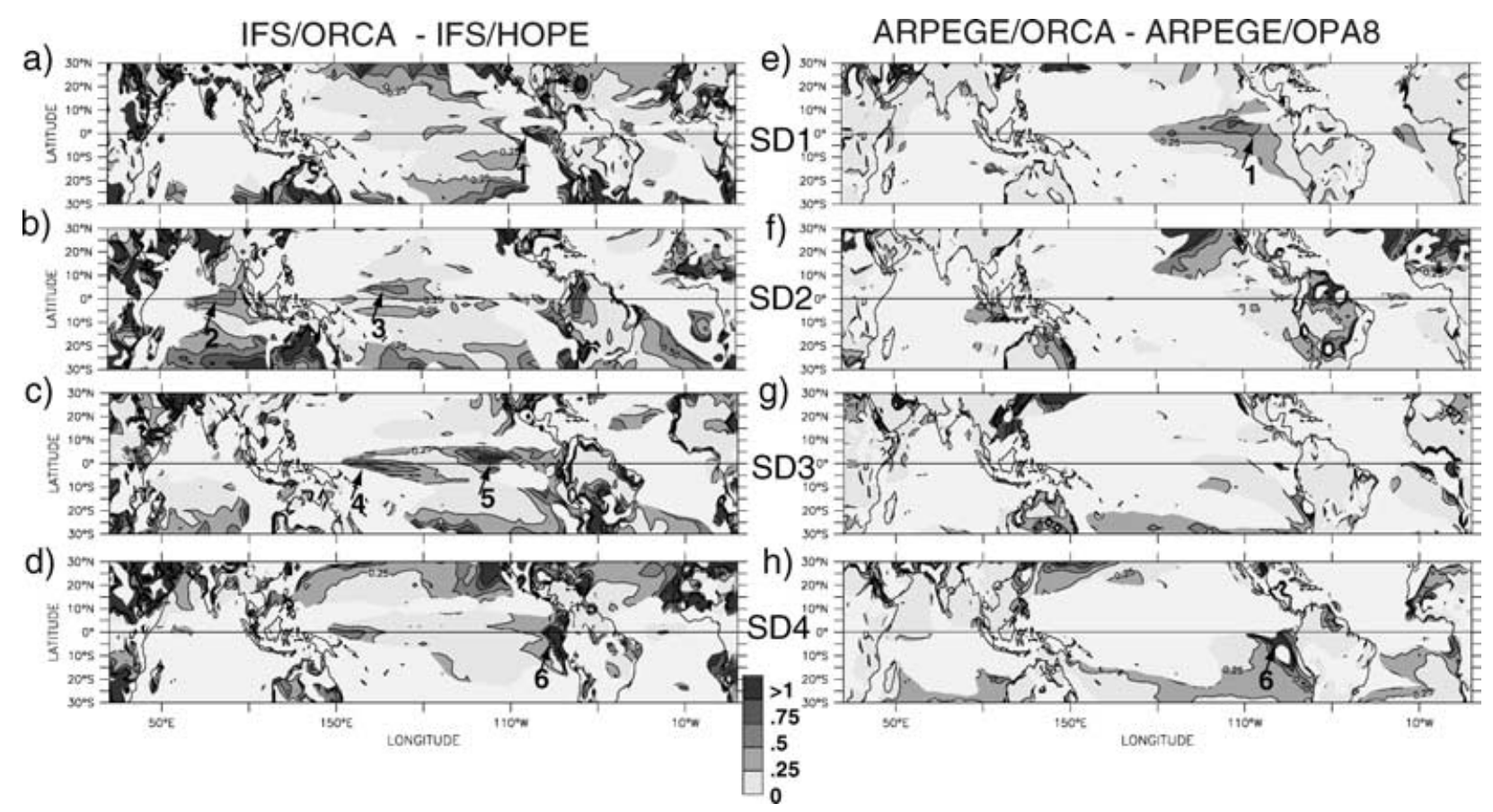

Fig. 3. Modification of the drift term after a change in the ocean component, relative to the average absolute drift term (see text), for each start date. Values are plotted only when one of the two absolute drift terms is larger than $1{ }^{\circ} \mathrm{C}$. Left column: $\mathrm{DCI}_{\mathrm{O}}$ (IFS/ORCA, IFS/HOPE). Right column: $\mathrm{DCI}_{\mathrm{O}}$ (ARPEGE/ORCA, ARPEGE/OPA8), contour interval 0.25. Arrows 1 to 6 indicate selected locations representative of the principal tropical areas of strong $\mathrm{DCI}_{\mathrm{O}}$.

$\mathrm{DCI}_{\mathrm{O}}$ values are much more frequently below 0.25 , indicating that the drift term is in general not strongly affected by change in the ocean component, as initially indicated by the equatorial domain averages.

Nonetheless this diagnostic only indirectly supports the idea, represented by eq. (1), that large increase in error depends mainly on the atmosphere component. One can indeed conceive that some of the error increases in Fig. 2 are weakly dependent upon a change of ocean component (low $\mathrm{DCI}_{\mathrm{O}}$ ), but are as weakly dependent upon a change of atmosphere component, simply because the major physical processes at play in the real world could be incorrectly simulated in all current CGCMs. It is particularly interesting to consider one of the less accurately simulated areas of the tropical oceans, namely the Atlantic (Davey et al. 2002, and Fig. 2, SD1)). We acknowledge the possibility that the error growth there, characterized by low $\mathrm{DCI}_{\mathrm{O}}$ in both pairs (see in particular Fig. 3, SD1), is mainly sensitive to the land processes and therefore not very sensitive to the ocean or atmosphere components of the CGCM. To address this remaining ambiguity we computed the following DCI, comparing the impact of a change in the ocean component to a change in the atmosphere component:

$$
\begin{aligned}
\mathrm{DCI}_{\mathrm{O} / \mathrm{A}}= & \mid\left(\text { Drift }_{\mathrm{OGCM} 1}-\text { Drift }_{\mathrm{OGCM} 2}\right) / \\
& \left(\text { Drift }_{\mathrm{IFS} / \mathrm{ORCA}}-\text { Drift }_{\mathrm{ARPEGE} / \text { ORCA }}\right) \mid
\end{aligned}
$$

Note that the numerator is the same as for $\mathrm{DCI}_{\mathrm{O}}$, but it is normalized by the only drift modification associated with an available change of atmosphere component. Indeed, in this set of experiments only ORCA is coupled to two AGCMs (IFS and ARPEGE). Hence, if the $\mathrm{DCI}_{\mathrm{O} / \mathrm{A}}$ is close to or greater than 1 , it suggests that changing the ocean component has as much, or more, impact on the error than changing the atmosphere component. The opposite applies when $\mathrm{DCI}_{\mathrm{O} / \mathrm{A}}$ is less than 1 . In the latter case, one must also consider that this could be a result of large impacts due to changes in the parametrization of land processes, which differs between the ARPEGE-based CGCMs and the IFS-based CGCMs. Consequently, in order to use this second DCI diagnostic to complement the interpretation of low $\mathrm{DCI}_{O}$ patterns, we shall consider the impact of the atmosphere/land component versus the ocean component on the error. Note once again that differences in the coupling procedure could potentially lead to changes in the drift term. Since at this stage we are focusing on areas with low values of the numerator (low $\mathrm{DCI}_{\mathrm{O}}$ ), it is the numerator that could be enlarged by these differences. Regions of low $\mathrm{DCI}_{\mathrm{O} / \mathrm{A}}$ should consequently not be considered. This is a limitation that does not impair our analysis since we are interested in detecting cases of $\mathrm{DCI}_{\mathrm{O} / \mathrm{A}}$ greater than or equal to 1 with a low $\mathrm{DCI}_{\mathrm{O}}$. These cases correspond to a change in the atmosphere or an ocean component which weakly modifies the 5-month error growth. In such cases, eq. (1) is less likely to be representative of the mean error behaviour. $\mathrm{DCI}_{\mathrm{O} / \mathrm{A}}$ values close to or greater than 1 in Fig. 4 point to a large number of locations, but when areas with high $\mathrm{DCI}_{\mathrm{O}}$ are ruled out only a 
(IFS/ORCA - IFS/HOPE) / (IFS/ORCA - ARPEGE/ORCA)

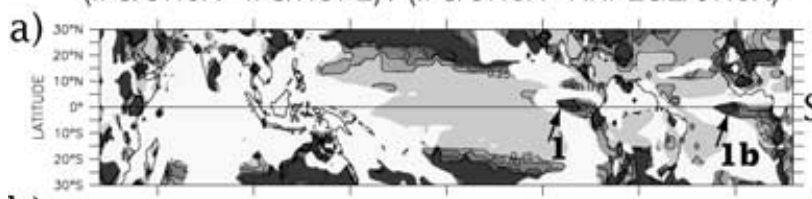

(ARPEGE/ORCA - ARPEGE/OPAB) / (IFS/ORCA - ARPEGE/ORCA)

b)
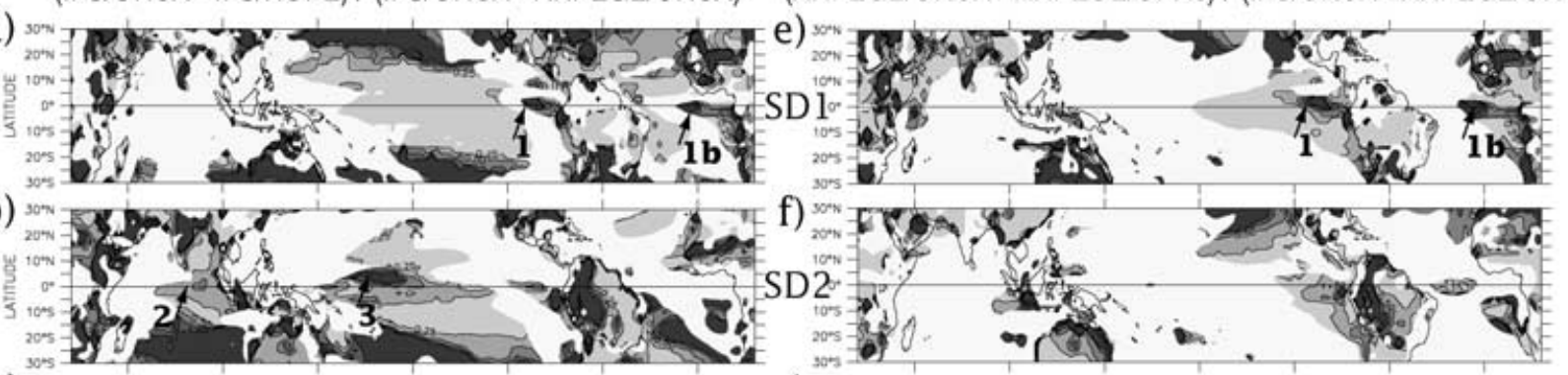

c)

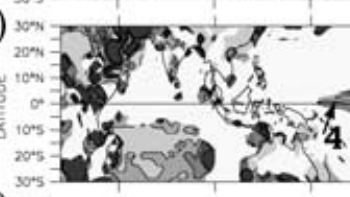

d)
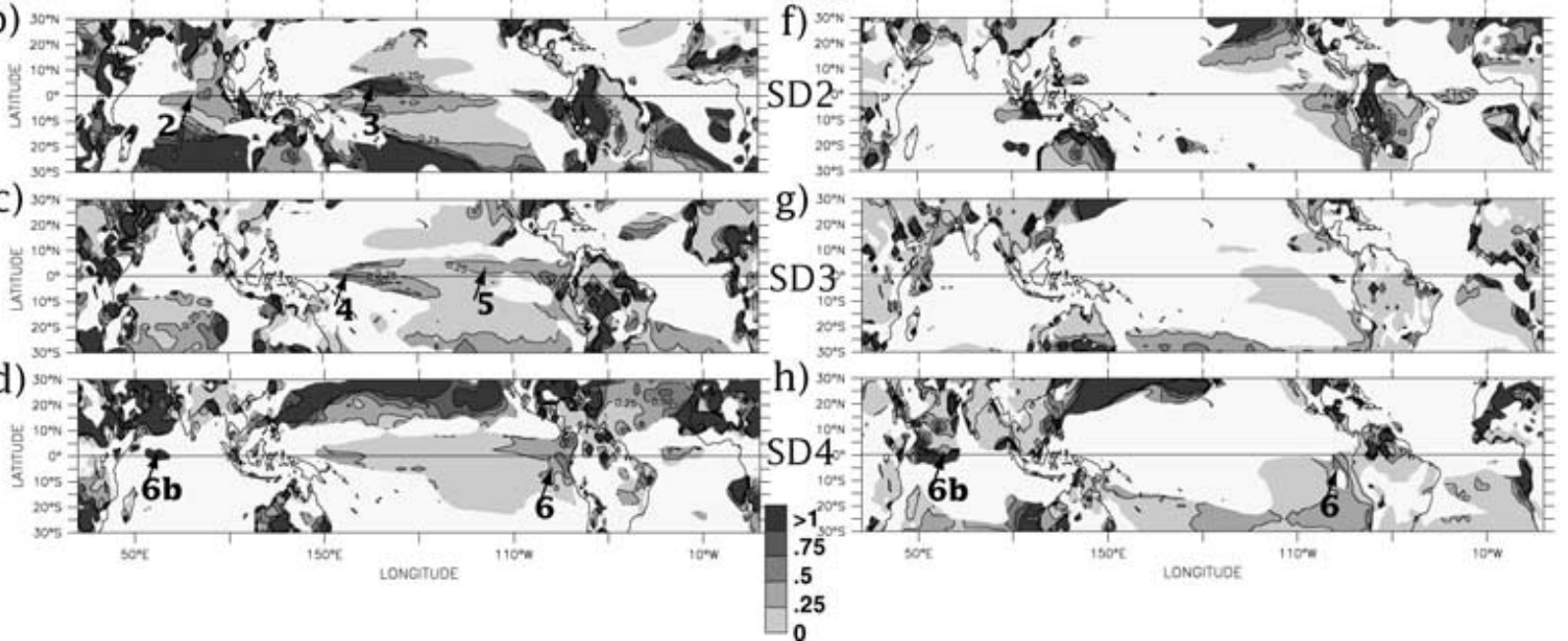

Fig. 4. Modification of the drift term after a change in the ocean component, relative to the change in drift term after a change in the atmosphere component (see text), for each start date. Values are plotted only when the absolute drift term is larger than $1{ }^{\circ} \mathrm{C}$. Left column: DCI $\mathrm{O} / \mathrm{A}$ (IFS/ORCA, IFS/HOPE). Right column: DCI $\mathrm{O}_{\mathrm{A}}$ (ARPEGE/ORCA, ARPEGE/OPA8), contour interval 0.25. Same arrows as Fig. 4, plus arrows $1 \mathrm{~b}$ and 6b indicating representative remaining locations of strong $\mathrm{DCI}_{\mathrm{O} / \mathrm{A}}$.

few regions remain. Within the tropical band, these regions are principally located over portions of the eastern equatorial $\mathrm{Pa}$ cific (Fig. 4e, arrow 1) and Atlantic (Figs 4a and e, arrow 1b) oceans for SD1, parts of the central Pacific (Fig. 4b, arrow 3) and south-tropical Atlantic oceans for SD2, and a small area of the equatorial Indian Ocean (Figs $4 \mathrm{~d}$ and $\mathrm{h}$, arrow $6 \mathrm{~b}$ ) for SD4. In order to further understand the mean error response to ocean and atmosphere components, these areas will also be examined in the following.

Successive inspection of the two DCI diagnostics indicated that most of the large growth in 5-month mean error visible in Fig. 2 is not strongly dependent on a change of ocean component. Locations where, on the contrary, this drift seems to be dependent on a change of ocean component, and others where this drift is similarly weakly dependent on a change in atmosphere and ocean component, are also apparent. The time evolution of the mean error for representative points of these areas is presented in Fig. 5. The existence of two types of time-series is immediately evident. One is made of time-series with a small spread among the four CGCMs. They correspond to the eastern Pacific and Central Atlantic locations for SD1, which corresponds to the season of development of upwelling (e.g. Li and Philander, 1996), and the West Indian location, for SD4, (Figs 5a, e and d). A second group is made of time-series which display a large spread, and more often for the IFS-based models: the Central Indian and western Pacific locations for SD2, and the western Pacific location for SD3 (Figs 5b, c and f).
The first group corresponds to time-series where the atmosphere appears to determine the details of the time evolution, but the ocean component takes 1 to 2 months for its impact on the error to settle, eventually becoming a constant shift. Equation (1) can still represent this type of behaviour rather well if one substitutes the mean error for the second month for that for the first month in the equation (i.e. the $\mathrm{ME}_{\text {month1 }}$ term would become $\left.\mathrm{ME}_{\text {month2 }}\right)$. However, as indicated by the high $\mathrm{DCI}_{\mathrm{O} / \mathrm{A}}$ values, the growth in the error after 6 months relative to month 1 , for a given location, is very similar regardless of the ocean and atmosphere component. This suggests that some important physical processes are missing in the CGCMs, due for example to parametrizations of ocean (e.g. upwelling mixing), atmosphere (e.g. stratocumulus cloud cover) or land processes. In the second group, the large spread of the errors for the IFS pair of models shows that the mean error at these locations and start dates does not follow eq. (1), as pointed out by the two DCI diagnostics. The switch in the ocean component is able to modify the growth in the error, and by as much as the change of atmosphere component. Although the drift term is not as large for the ARPEGE pair, the choice of ocean component is as important in the western Pacific (not visible on Fig. 3 due to the $1{ }^{\circ} \mathrm{C}$ threshold). The two remaining cases are the central and eastern Pacific for SD3 and SD4, respectively. The large $\mathrm{DCI}_{\mathrm{O}}$ values (except for the ARPEGE pair at location 5) come from DCI computation at month 6, which cannot account for time-series with a sinusoidal character. This results in a small denominator overestimating the 
a) East. Pac. (loc, 1)

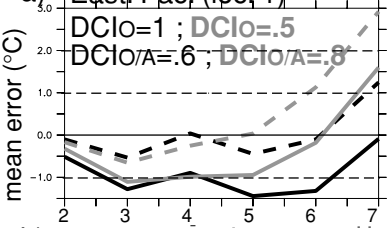

b) Cent. Ind. (loc, 2)

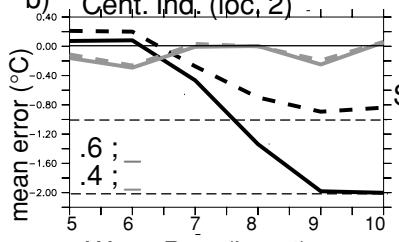

c) West. Pac. (loc. 4)

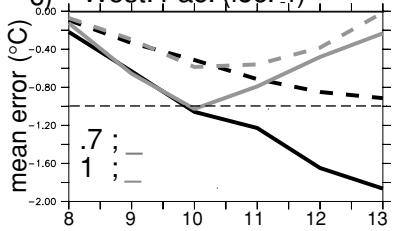

d) West. Ind. (loc. 6b)

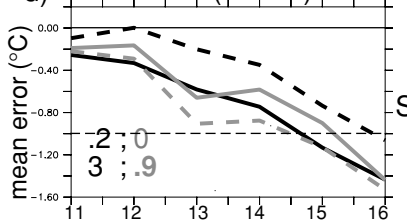

Calendar month

- IFS/ORCA e) Cent. Atl. (loc, 1b)
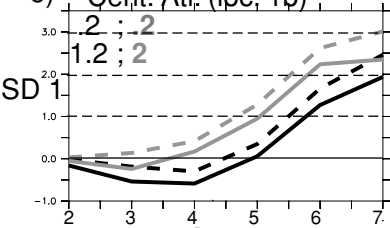

f) West. Pac. (loc. 3)

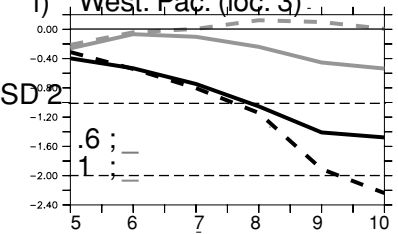

g) Cent. Pac. (Ioc. 5 )

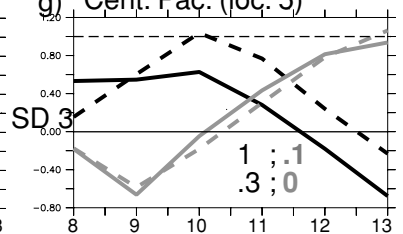

h) East. Pac. (loc. 6)

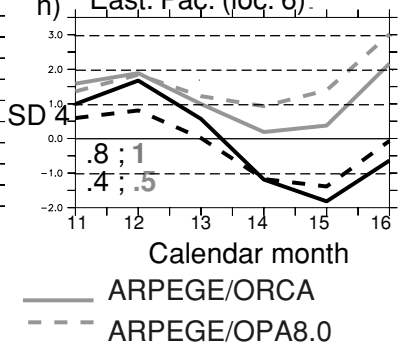

ARPEGE/OPA8.0

Fig. 5. Hindcast ensemble mean error and the two drift change indices for locations corresponding to the arrows on Fig. 4. Underscore stands for 5-month drifts smaller than $\pm 1^{\circ} \mathrm{C}$.

effect of a change in the ocean component in the time evolution of the error. The visual examination shows in fact that it is principally the atmosphere component that controls the error, and a change in the ocean component modulates it to second order after 1 to 2 months. In such a case however, eq. (1) is too simple to represent this effect. A detailed examination of the local mean error shows that these eight locations are representative of the corresponding eight areas that were highlighted by the two DCI analyses. We can therefore conclude that the strongest equatorial deviations to the behaviour represented by eq. (1) for the IFS-based CGCMs occur in the central Indian ocean for SD2 and over two limited areas in the Western Pacific, one for SD2 and one for SD3, visible in Fig. 3. The other areas, for which the error behaviour is represented by locations $1,1 \mathrm{~b}, 5,6$ and 7, are dominated by the atmosphere component with some influence from the ocean component. The latter is comparable in certain cases to a shift that is established in about 2 months, instead of one as initially proposed in eq. (1). Not surprisingly, the central and eastern equatorial Atlantic, the eastern Pacific and the western Indian oceans, experience a seasonal large drift that seems to be partly driven by common flaws in the physics of each of the models used.

\section{Summary and discussion}

The DEMETER project provides a multi-model ensemble of coupled simulations of the global climate for the past $30 \mathrm{yr}$. We analysed a subset of the hindcasts in terms of the sensitivity of the hindcast error to the atmosphere and the ocean components of the models, a component meaning the model itself and its initial conditions. The study, which focused on the tropical latitudes, was dedicated to the error in surface temperature averaged over ensemble hindcasts for the last decade. The important dependence of this mean error on the four hindcast start dates was also taken into account. Four CGCMs, forming two distinct atmosphere model pairs, were compared: the IFS AGCM was coupled to the ORCA and HOPE OGCMs, and the ARPEGE AGCM to the ORCA and the OPA8 OGCMs.

Examination of the equatorial basin averages of each coupled model suggested that the time evolution of the error, starting at month 1 , is very similar between CGCMs sharing the same atmosphere component. Generally, the change of the ocean component appears to merely result in a shift of the error curve by an almost constant value. Changing of atmosphere component, on the contrary, generally results in very different error behaviour. These results suggested a schematic representation of the error behaviour as the sum of a constant term and a time-evolving term. The former corresponds to the value of the error after the first month of coupling, and therefore includes the error in the initial condition. This term, corresponding to the apparent shift, is sensitive to both ocean and atmosphere components. The latter term, a time-dependent drift, is the remaining time-dependent error, which depends mainly upon the atmosphere component chosen and not the ocean one. Of course this is a simplified mathematical representation of the behaviour of the error. In fact, one can more precisely describe the latter as being formed of a rapidly evolving term depending essentially on the atmosphere component chosen and a slowly evolving term associated with climate processes with a larger timescale, associated naturally with the ocean and, probably also, with the slower atmospheric processes.

This decomposition of the mean error into two terms was then tested with two point-to-point drift change indices. One was evaluating the 5-month change of the drift term consecutive to a switch in the ocean component, the other the change consecutive to a switch in the atmosphere component. These analyses, carried out over the whole tropical band, showed first that the proposed decomposition does not hold for small errors, which are equally sensitive to a change in ocean or atmosphere component. In most cases, the drift term for large errors (of absolute value larger than about $1{ }^{\circ} \mathrm{C}$ ) instead displays a weak sensitivity to a change in ocean component. A small fraction of these areas of rapidly growing error would not follow this rule though, depending on the season. An additional test of the drift term indicated that in several regions it was neither sensitive to the ocean nor to the 
atmosphere. This recalls the known existence of missing physical processes (in the ocean, the atmosphere or the continent) in all the current models. The examination of local error time-series, for a sample of locations and start dates characterized by large changes in one or both drift change indices, allowed us to better describe the main exceptions to the decomposition initially proposed. The cases where the choice of the ocean component controls the rate of growth of the error as much as the atmosphere component occur over limited fractions of the Pacific warm pool and happen during the warming season (September to December) for all models, although the growth rate is smaller for the ARPEGEbased models. They also occur for all models in the eastern Pacific, over small areas and seasonally. In the west, one can speculate that the choice of the ocean component can affect the rate of growth of the error due to the sensitivity of the atmosphere to the high temperature of the thick mixed layer. This mechanism seems to be active in all models and is probably robust. It is likely that the large horizontal gradients associated with the upwelling are able to influence the time evolution of the error in the east. This is also a robust phenomenon. Finally, the drift in the central Indian Ocean for the IFS-based CGCMs is also sensitive to the ocean component, although it is very specific to the IFS-based models.

It is natural to wonder whether it is possible to anticipate the large error growth controlled by the atmosphere component as a function of the atmosphere model biases in forced mode. It is also possible that these large error growth rates only appear when the AGCM is coupled to an ocean model. In the first case, the improvement in the forecast drift would depend mainly on correcting the errors of the AGCM in forced mode. In the second case the task is more difficult, since the consequences of a change in a given atmospheric parametrization are much more difficult to anticipate and understand in the coupled system. In particular, the cold drift of the IFS-based models is consistent with the known cold biases of the AGCM that were attributed to atmospheric physics independently of the coupling with the ocean (Anderson et al., 2002). This supports the idea that large drifts in the coupled forecasts can be anticipated in most of the oceanic tropical areas. It is essential though to recall that our results are specific to the framework of seasonal forecasting, and they are probably model dependent. For example, one would like to look at the sensitivity of the error to the ocean component with an ocean model using isopycnic coordinates. Also, considering a longer timescale or higher latitudes would probably increase the impact of the ocean component. Further interpretation of the results of this paper in terms of the physics of ocean-atmosphere interactions is tempting but problematic. For example, the control of the error growth rate by the atmosphere could be considered to be a sign of a minor role for the ocean in the coupled system at this seasonal timescale in the tropics. But it is important to realize that it could also be interpreted as the mere consequence of the existence of much larger differences in the parametrizations among state of the art AGCMs than among state of the art OCGMs.

\section{Acknowledgments}

We wish to thanks A. S. Fisher, S. Janicot and P. Terray for stimulating discussions during this work.

\section{References}

AchutaRao, K. and Sperber, K. 2002. Simulation of the El Niño Southern Oscillation: results from the coupled model intercomparison project. Clim. Dynam. 19, 191-209.

Anderson, D., Stockdale, T., Balmaseda, M., Ferranti, L., Vitart, F. and co-authors, 2002. Comparison of the ECMWF seasonal forecast systems 1 and 2, including the relative performance for the 1997/98 El Niño. SAC Report, September 2002. ECMWF Research Department, Reading.

Davey, M., Huddleston, M., Sperber, K., Braconnot, P., Bryan, F. and co-authors, 2002. STOIC, a study of coupled model climatology and variability in tropical ocean regions. Clim. Dynam. 18, 403-420.

Delecluse, P., Davey, M., Kitamura, Y., Philander, S., Suarez, M. and co-authors, 1998. TOGA review paper: coupled general circulation modeling of the tropical Pacific. J. Geophys. Res. 103, 14357-14 373.

Gent, P. R. and McWilliams, J. C. 1990. Isopycnal mixing in ocean circulation models. J. Phys. Oceanogr. 20, 150-155.

Guilyardi, E., Gualdi, S., Slingo, J., Navarra, A., Delecluse, P. and coauthors, 2004. Representing El Niño in coupled ocean-atmosphere GCMs: the dominant role of the atmospheric component. J. Climate 17(24), 4623-4629.

Ji, M., Kumar, A. and Leetmaa, A. 1994. An experimental coupled forecast system at the National Meteorological Center: some early results. Tellus 46A, 398-418.

Kirtman, B. P. 1997. Oceanic Rossby wave dynamics and the ENSO period in a coupled model. J. Climate 10(7), 1690-1704.

Latif, M., Sperber, K., Arblaster, J., Braconnot, P., Chen, D. and coauthors, 2001. ENSIP: the El Niño simulation intercomparison. Clim. Dynam. 18, 255-276.

Latif, M., Sterl, A., Maier-Reimer, E. and Junge, M. M. 1993. Climate variability in a coupled general circulation model. Part I: The tropical Pacific. J. Climate 6, 5-21.

Lengaigne, M., Madec, G., Menkes, C. and Alory, G. 2003. Impact of isopycnal mixing on the tropical ocean circulation, J. Geophys. Res. 108(C11), 3345, doi:10.1029/2002JC001704.

Li, T. and Philander, S. G. H. 1996. On the annual cycle of the Eastern Equatorial Pacific. J. Climate 9, 2986-2998.

Mechoso, C. R., Robertson, A., Barth, N., Davey, M. and co-authors, 1995. The seasonal cycle over the tropical Pacific in coupled oceanatmosphere general circulation models. Mon. Weather Rev. 123, 28252838.

Palmer, T. N., Alessandri, A., Andersen, U., Cantelaube, P., Davey, M. and co-authors, 2004. Development of a European multi-model ensemble system for seasonal to inter-annual prediction (DEMETER). Bull. Am. Meteorol. Soc. 85(6), 853-872.

Raynaud, S., Speich, S., Guilyardi, E. and Madec, G. 2000. Impact of the ocean lateral diffusion on the ENSO-like variability of a global coupled GCM. Geophys. Res. Lett. 27, 3041-3044. 
Rosati, A., Miyakoda, K. and Gudgel, R. 1997. The impact of ocean initial conditions on ENSO forecasting with a coupled model. Mon. Weather Rev. 125(5), 754-772.

Schneider, E. K., DeWitt, D. G., Rosati, A., Kirtman, B. P., Ji, L. and co-authors, 2003. Retrospective ENSO forecasts: sensitivity to atmospheric model and ocean resolution. Mon. Weather Rev. 131, 30383060.

Stockdale, T. N. 1997. Coupled ocean-atmosphere forecasts in the presence of climate drift. Mon. Weather Rev. 125, 809818.

Vialard, J., Vitart, F., Balsameda, M. A., Stockdale, T. N. and Anderson, D. L. T. 2005. An ensemble generation method for seasonal fore- casting with an ocean-atmosphere coupled model. Mon. Weather Rev. 133, 441-453.

Vintzileos, A., Delecluse, P. and Sadourny, R. 1999a. On the mechanisms in a tropical ocean-global atmosphere coupled general circulation model. Part I: Mean state and the seasonal cycle. Clim. Dynam. 15, 43-62.

Vintzileos, A., Delecluse, P. and Sadourny, R. 1999b. On the mechanisms in a tropical ocean-global atmosphere coupled general circulation model. Part II: Interannual variability and its relation to the seasonal cycle. Clim. Dynam. 15, 63-80.

Yang, X-Q. and Anderson, J. L. 2000. Correction of systematic errors in coupled GCM forecasts. J. Climate 13, 2072-2085. 